ELECTRONIC RESEARCH ANNOUNCEMENTS

OF THE AMERICAN MATHEMATICAL SOCIETY

Volume 5, Pages 108-111 (July 20, 1999)

S $1079-6762(99) 00068-2$

\title{
THICKNESS MEASURES FOR CANTOR SETS
}

\author{
S. ASTELS
}

(Communicated by Yitzhak Katznelson)

\begin{abstract}
For a fixed $k \geq 1$ let $C_{1}, \ldots, C_{k}$ be generalized Cantor sets. We examine various criteria under which $C_{1}+\cdots+C_{k}$ contains an interval. When these criteria do not hold, we give a lower bound for the Hausdorff dimension of $C_{1}+\cdots+C_{k}$. Our work will involve the development of two different types of thickness measures.
\end{abstract}

\section{INTRODUCTION}

We define a generalized Cantor set (henceforth known as a Cantor set) to be any set $C$ of real numbers of the form

$$
C=I \backslash \bigcup_{i \geq 1} O_{i}
$$

where $I$ is a finite closed interval and $\left\{O_{i} ; i \geq 1\right\}$ is a countable (finite or infinite) collection of disjoint open intervals contained in $I$. We may inductively define a tree $\mathcal{D}$ that will represent $C$. Let the root of the tree be the interval $I$. We say that $\{I\}$ is the zeroth level of the tree. Now suppose we have defined our tree up to the $n^{t h}$ level. We define the $(n+1)^{t h}$ level of the tree as follows. Let $I^{w}$ be an $n^{\text {th }}$ level vertex of our tree. Assume first that

$$
I^{w} \cap\left(\bigcup_{i \geq 1} O_{i}\right) \neq \emptyset
$$

Let $O_{I}$ be the interval in the set $\left\{O_{i} ; i \geq 1\right\}$ of least index which is contained in $I^{w}$, and let $I^{w 0}$ and $I^{w 1}$ be closed intervals with

$$
I^{w}=I^{w 0} \cup O_{I^{w}} \cup I^{w 1} .
$$

We let $I^{w 0}$ and $I^{w 1}$ be subvertices of $I^{w}$ in $\mathcal{D}$. If

$$
I^{w} \cap\left(\bigcup_{i \geq 1} O_{i}\right)=\emptyset
$$

then we set $I^{w 0}=I^{w}$ and let $I^{w 0}$ be a subvertex of $I^{w}$ in $\mathcal{D}$. We repeat this process for every vertex $I^{w}$ in the $n^{t h}$ level of $\mathcal{D}$. The $(n+1)^{t h}$ level of the tree is the set

Received by the editors March 15, 1999 and, in revised form, May 12, 1999.

1991 Mathematics Subject Classification. Primary 58F12; Secondary 28A78.

Key words and phrases. Cantor sets, sums of sets, Hausdorff dimension.

Research supported in part by the Natural Sciences and Engineering Research Council of Canada.

(C)1999 American Mathematical Society 
of vertices $I^{v}$ in $\mathcal{D}$ with $|v|=n+1$, where $|v|$ denotes the length of the word $v$. We continue this process inductively, creating the infinite tree $\mathcal{D}$. Note that

$$
\left\{O_{I^{w}} ; I^{w} \text { is a bridge of } \mathcal{D}\right\}=\left\{O_{i} ; i \geq 1\right\},
$$

hence

$$
C=\bigcap_{n=0}^{\infty}\left(\bigcup_{|w|=n} I^{w}\right) .
$$

Any tree with this property is said to be a derivation of the Cantor set $C$ from $I$. The intervals $I, I^{0}, \ldots$ are called bridges of the derivation, while the open intervals $O_{I}, O_{I^{0}}, \ldots$ are called gaps of $C$.

Cantor sets arise naturally in many areas of mathematical inquiry, including the examination of the Markoff spectrum and the study of the chaotic behavior of certain families of functions (see, for example, [2] and [4]). Of interest to us here is the following problem. Define the sum of sets $E_{1}, \ldots, E_{n}$ to be the set

$$
E_{1}+\cdots+E_{n}=\left\{e_{1}+\cdots+e_{n} ; e_{i} \in E_{i} \text { for } 1 \leq i \leq n\right\}
$$

For $k \geq 2$ let $C_{1}, \ldots, C_{k}$ be Cantor sets derived from $I_{1}, \ldots, I_{k}$ respectively. In this paper we discuss conditions under which $C_{1}+\cdots+C_{k}$ contains an interval. We also give bounds for the Hausdorff dimension of $C_{1}+\cdots+C_{k}$.

\section{THICKNESS}

Let $C$ be a Cantor set. We define the thickness of $C, \tau(C)$, to be infinity if $\left\{O_{i} ; i \geq 1\right\}$ is empty. Otherwise we put

$$
\tau(C)=\sup _{\mathcal{D}} \inf _{A \in \mathcal{D}} \min \left\{\frac{\left|A^{0}\right|}{\left|O_{A}\right|}, \frac{\left|A^{1}\right|}{\left|O_{A}\right|}\right\}
$$

where the supremum is over all derivations $\mathcal{D}$ of $C$ and the infimum is over all bridges $A$ of $\mathcal{D}$. It is not difficult to show that the supremum is always attained (see, for example, [1], Lemma 3.1).

In 1979 Sheldon Newhouse [3] proved the following result.

Theorem 2.1. Let $C_{1}$ and $C_{2}$ be Cantor sets derived from $I_{1}$ and $I_{2}$ respectively, with $\tau\left(C_{1}\right) \tau\left(C_{2}\right)>1$. Then either $I_{1} \cap I_{2}=\emptyset, C_{1}$ is contained in a gap of $C_{2}, C_{2}$ is contained in a gap of $C_{1}$ or $C_{1} \cap C_{2} \neq \emptyset$.

In fact, if Newhouse's proof is slightly altered, then we may replace the condition " $\tau\left(C_{1}\right) \tau\left(C_{2}\right)>1$ " in Theorem 2.1 with the weaker condition " $\tau\left(C_{1}\right) \tau\left(C_{2}\right) \geq 1$ ". This strengthened version of Theorem 2.1 has the following corollary.

Theorem 2.2. For $j=1$ or $j=2$ let $C_{j}$ be a Cantor set derived from $I_{j}$, with $O_{j}$ a gap of maximal size in $C_{j}$. Assume that

$$
\left|O_{1}\right| \leq\left|I_{2}\right| \text { and } \quad\left|O_{2}\right| \leq\left|I_{1}\right|
$$

If $\tau\left(C_{1}\right) \tau\left(C_{2}\right) \geq 1$, then $C_{1}+C_{2}=I_{1}+I_{2}$.

If $\tau\left(C_{1}\right) \tau\left(C_{2}\right)<1$, then the work of Newhouse does not yield any non-trivial results. This case was the main focus of the author in [1], where a best-possible lower bound for the thickness of a finite sum of Cantor sets was found.

For a Cantor set $C$ we define the normalized thickness of $C, \gamma(C)$, to be

$$
\gamma(C)=\frac{\tau(C)}{\tau(C)+1} .
$$


Theorem 2.3. Let $k$ be a positive integer and for $j=1,2, \ldots, k$ let $C_{j}$ be a Cantor set derived from $I_{j}$, with $O_{j}$ a gap of maximal size in $C_{j}$. Let $S_{\gamma}=\gamma\left(C_{1}\right)+\cdots+$ $\gamma\left(C_{k}\right)$.

1. If $S_{\gamma} \geq 1$, then $C_{1}+\cdots+C_{k}$ contains an interval. Otherwise $C_{1}+\cdots+C_{k}$ contains a Cantor set of thickness at least

$$
\frac{S_{\gamma}}{1-S_{\gamma}}
$$

2. If

$$
\begin{aligned}
& \qquad \begin{array}{l}
\left|I_{r+1}\right| \geq\left|O_{j}\right| \quad \text { for } r=1, \ldots, k-1 \text { and } j=1, \ldots, r, \\
\left|I_{1}\right|+\cdots+\left|I_{r}\right| \geq\left|O_{r+1}\right| \quad \text { for } r=1, \ldots, k-1
\end{array} \\
& \text { and } S_{\gamma} \geq 1 \text {, then } \\
& \qquad C_{1}+\cdots+C_{k}=I_{1}+\cdots+I_{k} .
\end{aligned}
$$

3. If (1) and (2) hold and $S_{\gamma}<1$, then

$$
\tau\left(C_{1}+\cdots+C_{k}\right) \geq \frac{S_{\gamma}}{1-S_{\gamma}} .
$$

Proof. See [1], Theorem 2.4.

Note that in the case $k=2$ the condition " $\gamma\left(C_{1}\right)+\gamma\left(C_{2}\right) \geq 1$ " is equivalent to the condition " $\tau\left(C_{1}\right) \tau\left(C_{2}\right) \geq 1$ ", hence Theorem 2.3 implies Theorem 2.2.

Let $\operatorname{dim}_{H}(E)$ denote the Hausdorff dimension of the set $E$. There is a connection between thickness and Hausdorff dimension, as illustrated by the next theorem.

Theorem 2.4. If $C$ is a Cantor set, then

$$
\operatorname{dim}_{H}(C) \geq \frac{\log 2}{\log \left(2+\frac{1}{\tau(C)}\right)} .
$$

Proof. See [4], p. 77.

Using Theorem 2.3 and Theorem 2.4 we can establish the following lower bound for the Hausdorff dimension of a sum of Cantor sets.

Theorem 2.5. For $k \in \mathbb{Z}^{+}$let $C_{1}, \ldots, C_{k}$ be Cantor sets. Then

$$
\operatorname{dim}_{H}\left(C_{1}+\cdots+C_{k}\right) \geq \frac{\log 2}{\log \left(1+\frac{1}{\min \left\{\gamma\left(C_{1}\right)+\cdots+\gamma\left(C_{k}\right), 1\right\}}\right)} .
$$

It may be the case that $C_{1}+\cdots+C_{k}$ contains an interval yet $\gamma\left(C_{1}\right)+\cdots+\gamma\left(C_{k}\right)<$ 1. In this case better results may be gained by employing a concept known as maximal thickness.

\section{Maximal thiCKNESS}

We define the maximal thickness and normalized maximal thickness of a Cantor set $C$ to be

$$
\tau_{M}(C)=\sup _{C^{\prime} \subseteq C} \tau\left(C^{\prime}\right) \text { and } \gamma_{M}(C)=\frac{\tau_{M}(C)}{\tau_{M}(C)+1}
$$

respectively, where the supremum is taken over all Cantor sets $C^{\prime}$ contained in $C$. Note that for any Cantor set $C$ it follows trivially that $\tau(C) \leq \tau_{M}(C)$. Using Theorems 2.3 and 2.5 we may establish the following result. 
Theorem 3.1. Let $C_{1}, \ldots, C_{k}$ be Cantor sets. If

$$
\gamma_{M}\left(C_{1}\right)+\cdots+\gamma_{M}\left(C_{k}\right)>1
$$

then $C_{1}+\cdots+C_{k}$ contains an interval. Otherwise

$$
\gamma_{M}\left(C_{1}+\cdots+C_{k}\right) \geq \gamma_{M}\left(C_{1}\right)+\cdots+\gamma_{M}\left(C_{k}\right)
$$

and

$$
\operatorname{dim}_{H}\left(C_{1}+\cdots+C_{k}\right) \geq \frac{\log 2}{\log \left(1+\frac{1}{\gamma_{M}\left(C_{1}\right)+\cdots+\gamma_{M}\left(C_{k}\right)}\right)}
$$

\section{REFERENCES}

[1] S. Astels. Cantor sets and numbers with restricted partial quotients, Trans. Amer. Math. Soc. (to appear).

[2] Thomas W. Cusick and Mary E. Flahive. The Markoff and Lagrange spectra, Amer. Math. Soc., Providence, RI, 1989. MR 90i:11069

[3] Sheldon E. Newhouse. The abundance of wild hyperbolic sets and non-smooth stable sets for diffeomorphisms, Inst. Hautes Études Sci. Publ. Math. 50 (1979), 101-151. MR 82e:58067

[4] J. Palis and F. Takens. Hyperbolicity and sensitive chaotic dynamics at homoclinic bifurcations, Cambridge University Press, Cambridge, 1993. MR 94h:58129

Department of Pure Mathematics, The University of Waterloo, Waterloo, Ontario, CANADA N2L 3G1

E-mail address: sastels@barrow. uwaterloo.ca 